\title{
Malformação capilar jugal $x$ trauma parafuncional: relato de caso e tratamento
}

Capillary malformation x parafunctional trauma: case report and treatment
Malformación capilar $x$ trauma parafuncional: reporte de caso y tratamiento

Juliana dos Santos CATIVO

Lioney Nobre CABRAL

Tiago Novaes PINHEIRO

Myrian Salles VIEIRA

Antonio Jorge de Araujo VASCONCELOS II

Escola de Ciências da Saúde, Universidade do Estado do Amazonas (ESA-UEA) Cachoeirinha - 69065.001 Manaus-AM, Brasil

\begin{abstract}
Resumo
As malformações vasculares são anomalias estruturais dos vasos sanguíneos sem proliferação endotelial, estão presentes ao nascimento e persistem ao longo da vida. Elas podem ser categorizadas de acordo com o tipo de vaso envolvido (capilar, venoso, arteriovenoso) e de acordo com as características hemodinâmicas (baixo fluxo ou alto fluxo). Possuem uma etiologia variável, como fatores químicos, mutações genéticas ou alterações relacionadas a síndromes, fluxo sanguíneo, resistência da parede vascular e trauma. Neste caso, a malformação vascular apresentada pela paciente tem relação com o trauma em mucosa jugal devido a atividade parafuncional presente. Atividade parafuncional refere-se a qualquer atividade que não seja considerada funcional, como apertar e/ou ranger os dentes durante o dia e/ou à noite, mascar chicletes, morder bochecha, lábios e língua. Algumas destas atividades podem ser responsáveis por concorrer para a Disfunção Temporomandibular (DTM), pois promovem hiperatividade muscular mastigatória. Dor muscular, restrição de abertura de boca, sensação de plenitude auricular, zumbido na orelha e vertigem foram alguns dos sintomas apresentados pela paciente. Diante desse quadro, além de tratar a malformação vascular com uma combinação de escleroterapia e excisão cirúrgica, foi também tratado o quadro disfuncional, para seu controle e resolução além da prevenção de recidiva da lesão, com a utilização de placa miorrelaxante combinada com a realização de exercícios fisioterápicos, mostrando-se eficazes, contendo a sintomatologia, restringindo a atividade parafuncional e reduzindo o trauma na mucosa.
\end{abstract}

Descritores: Malformação vascular; Escleroterapia; Transtornos da Articulação Temporomandibular; Placas Oclusais.

\section{Abstract}

Vascular malformations are structural abnormalities of blood vessels without endothelial proliferation, they are at birthday and persist throughout life. They can be categorized according to the type of vessel involved (capillary venous, arteriovenous) and according to hemodynamic characteristics (low flow or high flow). They have a variable etiology, such as chemical factors, genetic mutations or changes related to syndromes, blood flow, vascular body resistance and trauma. In this case, the vascular malformation presented by the patient is related to trauma to the cheek mucosa due to the parafunctional activity present. Parafunctional activity refers to any activity that is not considered to be functional, such as clenching and / or grinding your teeth during the day and / or at night, chewing gum, biting your cheek, lips and tongue. Some of these activities may be responsible for contributing to Temporomandibular Dysfunction (TMD), as they promote masticatory muscle hyperactivity. Muscle pain, restriction of mouth opening, sensation of ear fullness, ringing in the ear and vertigo were some of the symptoms presented by the patient. In view of this condition, in addition to treating vascular malformation with a combination of sclerotherapy and surgical excision, the dysfunctional condition was also treated, for its control and resolution, in addition to preventing injury recurrence, with the use of myorelaxant plaque combined with the performance of physical therapy exercises, proving to be effective, containing symptoms, restricting parafunctional activity and reducing trauma to the mucosa.

Descriptors: Vascular malformation; Sclerotherapy; Temporomandibular Joint Disorders; Occlusal Splints.

\section{Resumen}

Las malformaciones vasculares son anormalidades estructurales de los vasos sanguíneos sin proliferación endotelial, están presentes al nacer y persisten durante toda la vida. Se pueden clasificar según el tipo de vaso involucrado (capilar, venoso, arteriovenoso) y según las características hemodinámicas (flujo bajo o flujo alto). Tienen una etiología variable, como factores químicos, mutaciones genéticas o cambios relacionados con síndromes, flujo sanguíneo, resistencia de la pared vascular y traumatismos. En este caso, la malformación vascular presentada por el paciente está relacionada con un traumatismo en la mucosa de la mejilla debido a la actividad parafuncional presente. La actividad parafuncional se refiere a cualquier actividad que no se considere funcional, como apretar y rechinar los dientes durante el día y / o la noche, masticar chicle, morderse la mejilla, los labios y la lengua. Algunas de estas actividades pueden ser responsables de contribuir a la disfunción temporomandibular (TMD), ya que promueven la hiperactividad del músculo masticatorio. Dolor muscular, restricción de la apertura de la boca, sensación de llenura del oído, zumbido en el oído y vértigo fueron algunos de los síntomas presentados por el paciente. En vista de esta condición, además de tratar la malformación vascular con una combinación de escleroterapia y escisión quirúrgica, la condición disfuncional también se trató, para su control y resolución, además de prevenir la recurrencia de lesiones, con el uso de placa miorrelajante combinada con la realización de ejercicios fisioterapéuticos, que demuestran ser efectivos, que contienen síntomas, restringen la actividad parafuncional y reducen el trauma en la mucosa. Descriptores: Malformación vascular; Escleroterapia; Trastornos de la articulación temporomandibular; Férulas Oclusales.

INTRODUÇÃO

A distinção entre hemangiomas e malformações vasculares foi desconhecida por um longo período de tempo. Os hamartomas vasculares, dos quais os hemangiomas são o principal representante, são verdadeiras neoplasias dos vasos sanguíneos, formados por proliferação endotelial assumindo largura variável. A lesão geralmente está presente no nascimento e pode regredir espontaneamente, ao contrário das malformações vasculares que não exibem ativa proliferação das células endoteliais e podem persistir durante a vida ${ }^{1,2}$.

As malformações vasculares consistem em um aumento progressivo dos vasos, compostos por uma arquitetura vascular como veias, vasos linfáticos, vênulas, capilares, artérias ou tipos de vasos mistos. Elas podem ser divididas em malformações de alto e baixo fluxo: malformações arteriovenosas são alto fluxo devido ao padrão de fluxo sanguíneo 
arterial, malformações capilares, linfáticas e venosas são lesões de baixo fluxo. A maior proporção de malformações de baixo fluxo é localizada na região da cabeça e pescoço ${ }^{3-5}$.

Clinicamente as malformações vasculares variam de acordo com a extensão da lesão, localização e deslocamento de tecido vizinho. Elas podem ser isoladas ou múltiplas e também fazer parte de outras doenças sistêmicas ${ }^{2}$.

O exame clínico e manobras semiotécnicas como a diascopia ou vitropressão são as principais formas de realizar 0 diagnóstico de malformações vasculares ${ }^{6}$. Além disso, exames de imagem como radiografias, tomografias e ressonância magnética, concorrem para a restrição anatômica e também possibilitam a determinação de diagnósticos diferenciais, ou para detectar a presença de anomalias associadas, auxiliando na definição do tratamento ${ }^{7,8}$. A abordagem multidisciplinar é necessária não somente para o diagnóstico, mas também para 0 tratamento das malformações vasculares ${ }^{3}$.

O tratamento a ser indicado para as malformações vasculares é dependente da idade do paciente, do tamanho das lesões e das características clínicas. As opções terapêuticas são diversas e incluem: medidas conservadoras como elevação e compressão da cabeceira da cama, laserterapia, escleroterapia, embolização e cirurgia ${ }^{2}$. Pequenas malformações estáveis podem não requerer tratamento. Lesões grandes e problemáticas podem ser tratadas com uma combinação de escleroterapia e excisão cirúrgica ${ }^{1}$.

No presente caso, a malformação vascular capilar apresentada pela paciente pode ter surgido devido ao trauma na mucosa decorrente da atividade parafuncional. Além disso, a paciente também apresentava um quadro de DTM. Sabe-se que as atividades parafuncionais podem ser um fator etiológico da DTM, assim como uma condição secundária.

A disfunção temporomandibular (DTM) é um termo que engloba uma série de disfunções e desordens que afetam a articulação temporomandibular (ATM) e/ou os músculos mastigatórios e estruturas associadas. Essas desordens se caracterizam por um conjunto de sinais e sintomas peculiares, que podem incluir ruídos articulares, como estalidos e crepitação, dor nos músculos mastigatórios, limitação dos movimentos mandibulares, dores faciais, dores de cabeça e dores na articulação temporomandibular, além de dores na região periauricular ${ }^{9}$.
O caráter da etiologia da DTM é multifatorial, no qual inúmeros fatores etiológicos podem contribuir para o aparecimento dos sinais e sintomas relacionados ao sistema estomatognático, devido à alteração da função natural deste sistema. Os fatores sistêmicos são aqueles capazes de diminuir a tolerância fisiológica do indivíduo a uma lesão ou à dor. Como exemplo, as interferências emocionais e afetivas na interpretação da dor e da disfunção, baixo condicionamento físico, má qualidade nutricional e do sono. Dentre os fatores locais estão a interferência oclusal, o traumatismo muscular ou articular e os hábitos parafuncionais ${ }^{10}$.

Atividade parafuncional refere-se a qualquer atividade que não seja considerada funcional (mastigação, fala e deglutição). Isto inclui o bruxismo, o apertamento dentário e certos hábitos orais. Algumas destas atividades podem ser responsáveis por criar sintomas de $\mathrm{DTM}^{11}$. Estas parafunções alteram o fluxo sanguíneo normal dos tecidos musculares, ocasionando acúmulo de produtos metabólicos nas células destes tecidos, desencadeando sintomas de fadiga, dor e espasmo ${ }^{10}$.

Como formas de tratamentos estão: educação do paciente, autocuidado, alteração de hábitos comportamentais, medicamentos, atividades fisioterápicas, placas oclusais estabilizadoras, terapia oclusal, tratamento ortodôntico, reabilitação oral, acupuntura, laserterapia, ondas de choques e cirurgia. $O$ tratamento conservador é o mais indicado para os casos iniciais de DTM, por apresentar bons resultados e não ser irreversível ${ }^{12}$.

Este trabalho tem como objetivo mostrar o tratamento de escolha para uma paciente que apresentava uma malformação vascular capilar e disfunção temporomandibular.

CASO CLÍNICO

Paciente leucoderma, 53 anos, sexo feminino procurou o serviço de estomatologia da Universidade do Estado do Amazonas queixando-se de uma "bolha" na bochecha direita. Ao exame clínico intraoral observou-se uma lesão na mucosa jugal direita ao nível do plano oclusal, esta, elevada, circular, de cor arroxeada, com cerca de $8 \mathrm{~mm}$, de consistência macia e assintomática a palpação (Figura 1). 


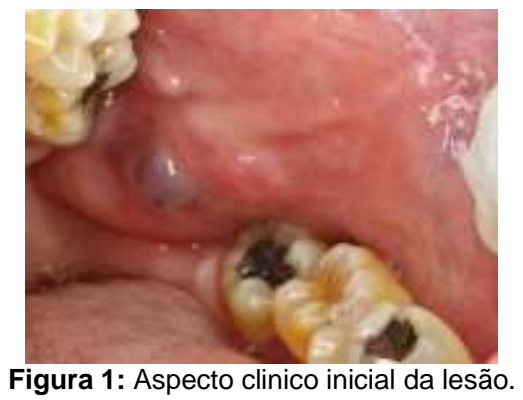

Realizou-se diascopia na lesão, onde notou-se o esmaecimento de sua coloração. $O$ diagnóstico presuntivo foi de malformação vascular ou hemangioma. $\mathrm{Na}$ anamnese a paciente relatou que tinha o hábito de mordiscar a bochecha, mas que não sabia dizer quando aconteceu o surgimento da lesão. Relatou não sentir dor ou sensação de calor, apenas incômodo ao mastigar alimentos. Quando questionada, a paciente relatou sentir vertigem, zumbido na orelha, sensação de plenitude auricular e dores de cabeça, afirmou também que havia a presença de ruído na articulação temporomandibular (ATM) durante a mastigação. A paciente revelou já ter feito um tratamento para DTM, mas que não deu continuidade e não houve melhora na sintomatologia. Ao exame clinico extraoral observou-se restrição na abertura de boca e durante a palpação a mesma apresentou pontos de gatilho em ambos os lados dos músculos temporal, masseter, esternocleidomastóideo, cervicais posteriores e trapézio descendente. Ao examinar a ATM percebeu-se a presença de estalido, crepitação na abertura e fechamento bucal. Considerando os fatos, optou-se pela escleroterapia como tratamento da lesão e o uso de uma placa oclusal miorrelaxante combinada com exercícios fisioterápicos para tratar o quadro de DTM e atividade parafuncional. Foi injetado $0,1 \mathrm{ml}$ de Oleato de Monoetanolamina a $5 \%$ (Ethamolin) após anestesia com Cloridrato de Prilocaína a 3\%, na base e na parte mais central da lesão. (Figuras 2 e 3) Após sete dias da injeção da solução a lesão diminuiu de tamanho, mas não completamente, como era 0 esperado e permaneceu sujeita a trauma. (Figura 4).

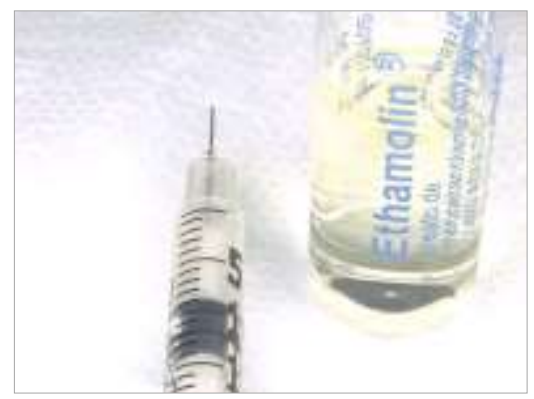

Figura 2: Injeção de Ethamolin.

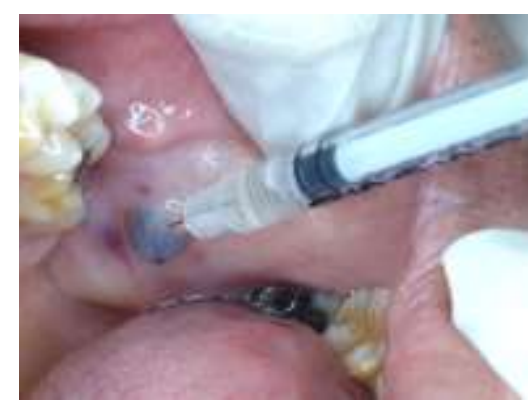

Figura 3: Injeção de Ethamolin.

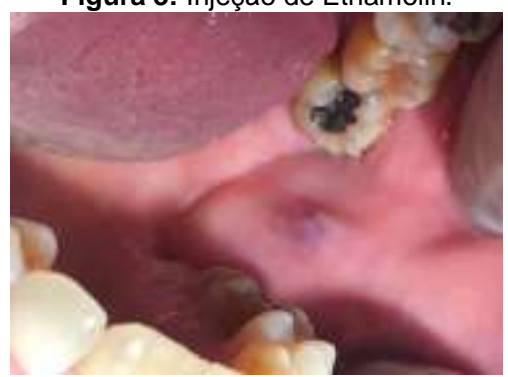

Figura 4: Aspecto da lesão após sete dias da injeção.

Para a confecção da placa miorrelaxante foi realizada a moldagem, registro do arco facial (Figura 5) e montagem dos modelos em articulador semi-ajustavel (ASA). Durante o ajuste e instalação da placa miorrelaxante (Figura 6 e 7) a paciente relatou que o trauma na lesão ficou mais acentuado, levando a opção de sua remoção completa por meio de biopsia excisional.
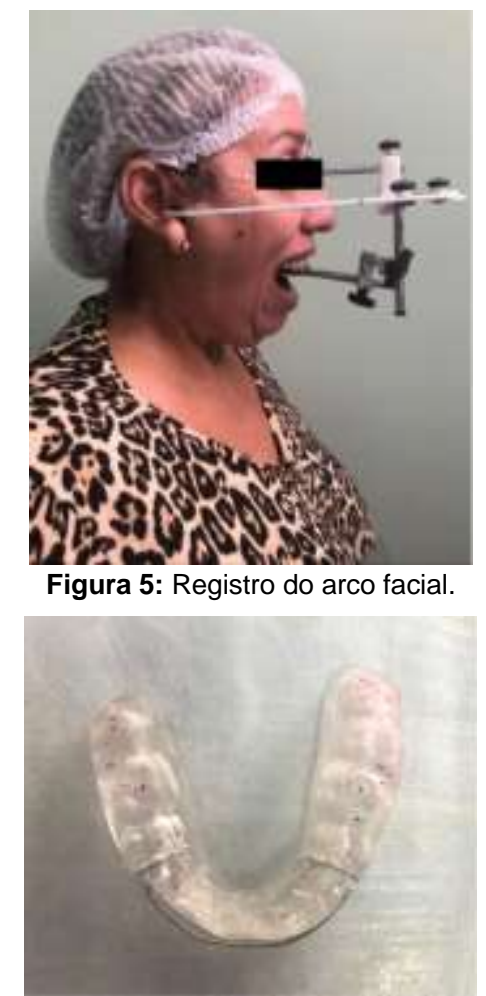

Figura 6: Ajuste da placa miorrelaxante. 


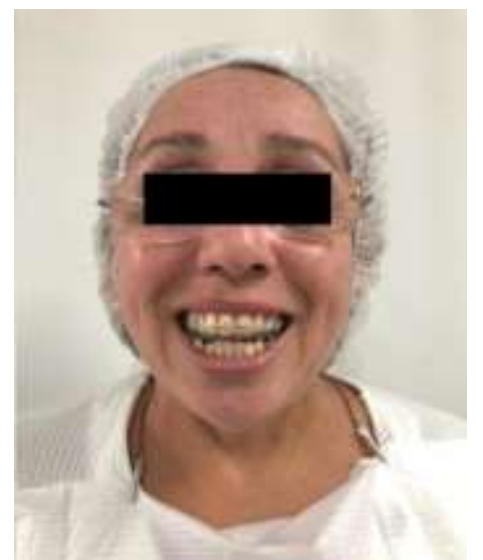

Figura 7: Instalação da placa miorrelaxante.

A excisão foi feita em ambulatório, sob anestesia local, sem complicações transoperatórias. (Figuras 8 a 10). Após sete dias foi realizada a remoção dos pontos (Figura 11). A paciente não relatou nenhum incomodo após a cirurgia e não se observou alterações na região.

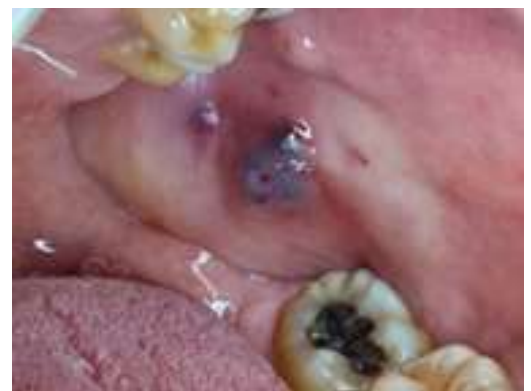

Figura 8: Aspecto da lesão após anestesia.

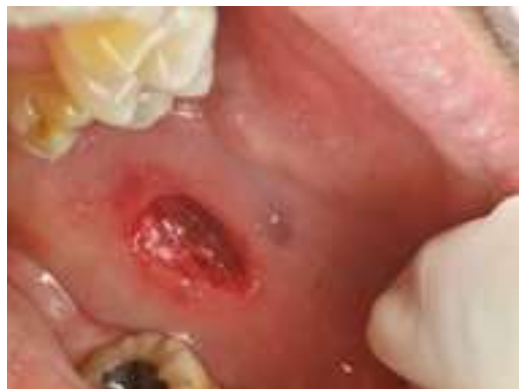

Figura 9: Aspecto da lesão após biópsia excisional.

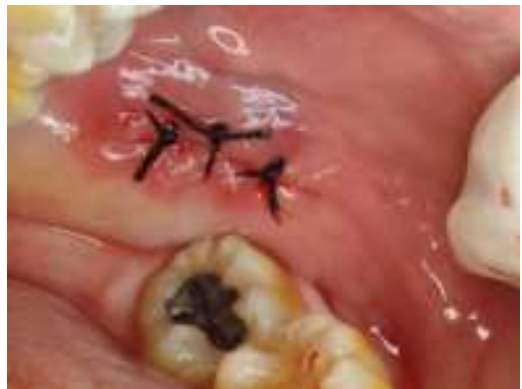

Figura 10: Síntese.

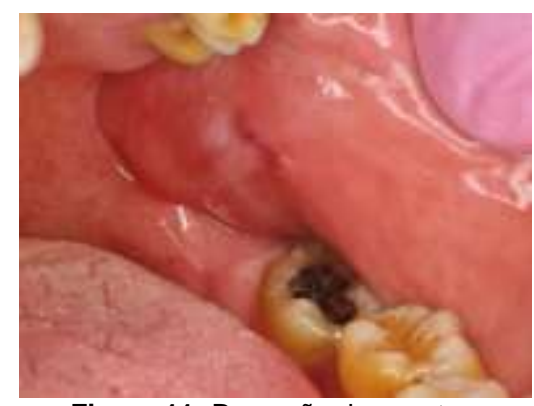

Figura 11: Remoção dos pontos.

Microscopicamente os cortes revelam fragmento de mucosa bucal revestido por epitélio bucal estratificado pavimentoso queratinizado hiperplásico. Subjacente o tecido conjuntivo é frouxo não modelado, apresenta área central rica em capilares congestos, e numa posição intermediaria mais profunda um vaso em reorganização pós tromboembolismo, celularizado por células endoteliais formando aglomerados epitelióides e capilares congestos. O diagnóstico foi de malformação vascular capilar e tromboembolismo em estado de revascularização. (Figura 12 e 13). Além do uso da placa miorrelaxante durante o dia, a paciente foi instruída a praticar os seguintes exercícios fisioterápicos: 1) Abertura de boca contra resistência, onde com as palmas das mãos apoiadas no queixo a paciente deve abrir a boca minimamente por dez segundos e fechar, repetir o movimento dez vezes; (Figura 14) 2) Abertura e fechamento orientado, onde os dedos indicador e polegar devem ficar entre os dentes e realizar abertura e fechamento de boca por dez vezes, três séries deste devem ser realizadas (Figura 15); 3) Abertura em maior amplitude, onde a paciente deve colocar a língua no céu da boca e tentar abri-la em sua maior amplitude por dez segundos, fechar e repetir o movimento três vezes (Figura 16).

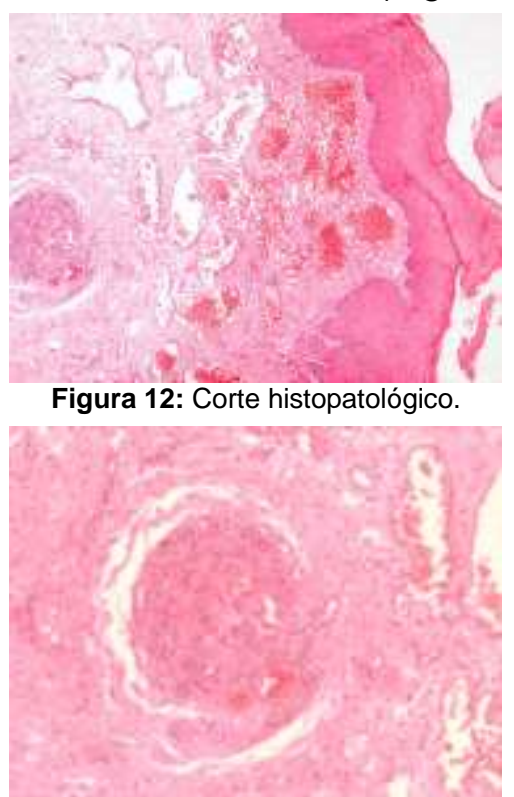

Figura 13: Corte Histopatológico. 


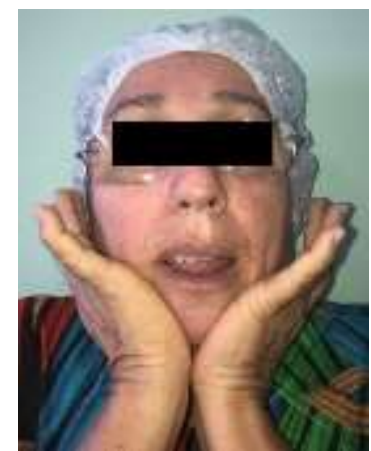

Figura 14: Exercício: Abertura de boca contra resistência.

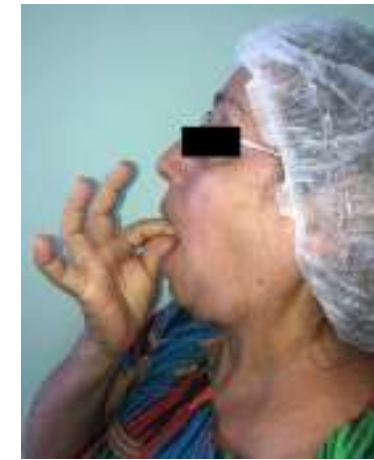

Figura 15: Exercício: Abertura e fechamento orientado

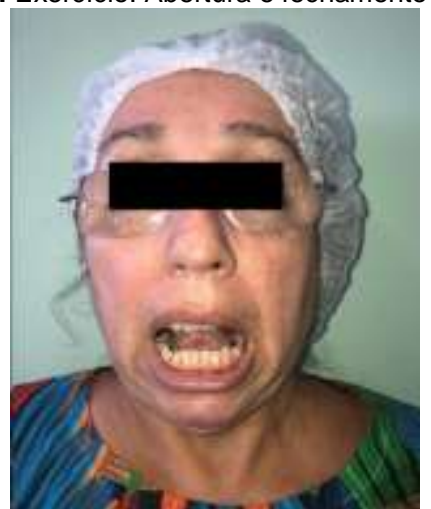

Figura 16: Exercício: Abertura em maior amplitude.

Foram marcados retornos semanais, a fim de se verificar os resultados do tratamento e fazer novos ajustes, caso necessário. Ao final do tratamento constatou-se que os tratamentos propostos foram eficazes, melhorando a restrição de abertura de boca (Figuras 17 e 18), restringindo a atividade parafuncional $e$ reduzindo o trauma na mucosa, além das dores apresentadas pela paciente, resultante dos pontos de gatilho nas musculatura mastigatória e cervical (Figura 19).

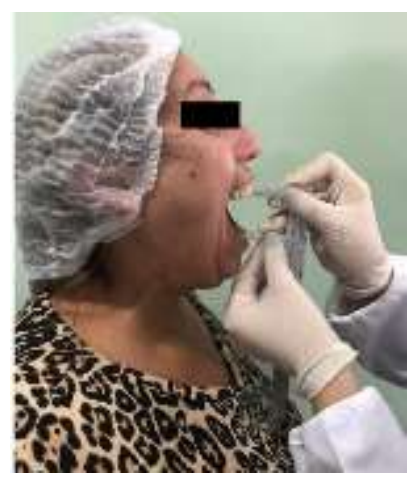

Figura 17: Abertura de boca inicial: $30 \mathrm{~mm}$.

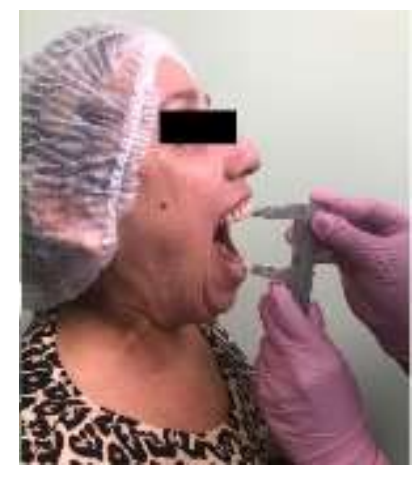

Figura 18: Abertura de boca final: $37 \mathrm{~mm}$.

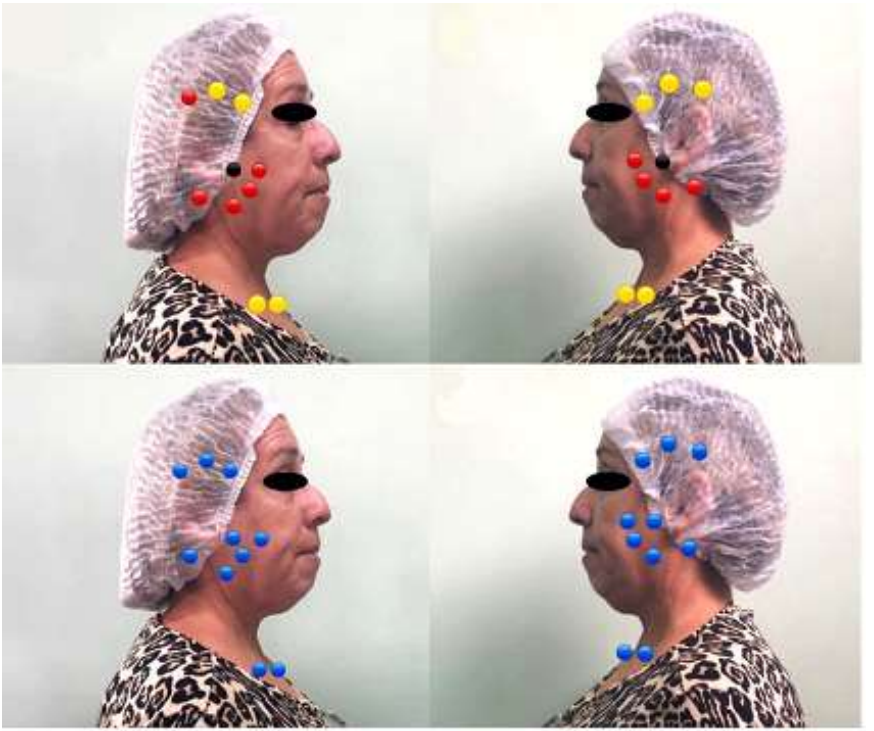

Figura 19: Avaliação dos pontos de gatilho inicial e final do lado direito e esquerdo. Azul: ausência de dor; Amarelo: Sensibilidade, Preto: dor; Vermelho: Reação de fuga.

DISCUSSÃO

Ao contrário dos hemangiomas, as malformações vasculares estão presentes ao nascimento e persistem ao longo da vida. As malformações capilares afetam principalmente a pele e mucosa, apresentando-se geralmente como máculas que a coloração varia de rosa a vermelho, chamadas de "manchas de vinho-doporto". Ocorrem em $0,3 \%$ a $1,0 \%$ dos recémnascidos. São mais comuns na face, particularmente ao longo da região inervada pelo nervo trigêmeo. Essas lesões podem engrossar e escurecer com o tempo e podem estar associadas a tecidos moles ou crescimento ósseo ${ }^{1,5}$. Clinicamente as malformações vasculares variam de acordo com à extensão da lesão, localização e deslocamento de tecido vizinho. Elas podem ser isoladas ou múltiplas e fazer parte de outras doenças sistêmicas².

A incorreta nomenclatura e erros de diagnóstico são comumente vivenciados pelos pacientes com anomalias vasculares ${ }^{5}$. O diagnóstico correto de malformações vasculares tem um papel importante no planejamento adequado do tratamento. A escolha de uma remoção cirúrgica, escleroterapia, embolização ou imunoterapia está ligada à classificação da lesão ${ }^{2}$. No presente caso a opção de escolha foi escleroterapia com oleato de monoetanolamina a $5 \%$ e posteriormente excisão cirúrgica.

A escleroterapia envolve injeção percutânea ou por mucosa de uma substância para induzir inflamação e trombose da lesão que levará a fibrose a longo prazo e diminuição 
parcial ou total da lesão. A escleroterapia préoperatória é benéfica, pois diminuiu 0 sangramento e facilitou a dissecação e, além disso, reduziu a perda de sangue e o tempo operatório. Em alguns casos, a cirurgia é uma garantia quando a ocorre falha na escleroterapia ${ }^{2}$, como no presente caso. A excisão cirúrgica é uma boa opção de tratamento e, na opinião dos autores, continua sendo a melhor chance de cura das malformações ${ }^{3}$.

A disfunção temporomandibular é apontada como a principal causa de dor de origem não dentária na região orofacial ${ }^{13}$. A mesma pode acometer indivíduos dentados e edentados, usuários ou não de próteses ${ }^{14}$.

Uma análise da literatura mostra que a DTM envolve mais as mulheres do que os homens, com uma razão que varia de 3:1: a 5:1, com uma variação média de idade entre 30 e 40 $\operatorname{anos}^{15}$.

Os objetivos do tratamento em pacientes com DTM incluem reduzir a dor, reduzir a sobrecarga adversa e restaurar a função, possibilitando ao paciente retomar as atividades diárias normais, assim, a associação de duas ou mais terapias pode ser indicada. Por ser uma disfunção com etiologia de caráter multifatorial, seu diagnóstico e tratamento são complexos, tendo uma variedade de opções, como, o uso de placas oclusais miorrelaxantes, exercícios fisioterápicos, laserterapia e acunputura ${ }^{12}$, no presente caso o tratamento de escolha para a DTM foi placa miorrelaxante e exercícios fisioterápicos.

Os exercícios terapêuticos têm sido muito empregados na reabilitação e prevenção da DTM, com o objetivo de aliviar a dor e melhorar a função, porém são escassos os trabalhos que comparem e discutam a eficácia dos mesmos ${ }^{16}$, por isso a importância desse estudo. A intervenção através de exercícios prioriza a musculatura mastigatória e da região cervical para melhora da força e a mobilidade da região ${ }^{17}$. Além disso, de uma forma geral, a fisioterapia estimula a propriocepção e a produção do líquido sinovial na articulação e melhora a elasticidade das fibras musculares aderidas $^{18}$.

Estudos mostram que o uso de placa associado a exercícios apresentaram resultados significativos ${ }^{17}$. A paciente desse caso fez o uso uma placa miorrelaxante superior, pois as placas estabilizadoras de resina acrílica melhoram as desarmonias e possíveis desvios dos movimentos mandibulares, reposicionam a mordida em relação cêntrica, favorecendo uma posição articular confortável, reposicionam o côndilo, relaxando a musculatura mastigatória e suavizando a dor ${ }^{12}$. Nesse caso houve uma melhora significativa nos sintomas da DTM e diminuição do hábito parafuncional.

É importante destacar que o sucesso do tratamento dependeu da responsabilidade da paciente em seguir as instruções que lhe foram repassadas. A sua dedicação em realizar diariamente os exercícios fisioterápicos e usar a placa miorrelaxante foram essenciais para o resultado satisfatório.

CONCLUSÃO

A escleroterapia pré-operatória e a excisão cirúrgica da lesão foram uma boa terapia de escolha. O uso de uma placa miorrelaxante combinada com a realização de exercícios fisioterápicos como terapia de escolha, mostrou-se eficaz, contendo a sintomatologia, em destaque as dores apresentadas pela paciente, resultante dos pontos de gatilho nas musculaturas mastigatória e cervical. Além disso, foram eficazes restringindo a atividade parafuncional $e$ reduzindo o trauma na mucosa, prevenindo assim a recidiva da lesão.

\section{REFERENNCIAS}

1. Neville BW, Damm DD, Allen CM, Bouquot JE. Patologia Oral e Maxilofacial. 3 ed. Rio de Janeiro: Elsevier; 2009.

2. Kuriakose MA, Chakrabarti S, Cheong SC, Kowalski LP, Novaes Pinheiro T, Farah CS. Head and neck tumors. In: Farah C, Balasubramaniam $\mathrm{R}$, McCullough $\mathrm{M}$ (eds). Contemporary oral medicine. Springer. Cham; 2019.

3. Buckmiller LM, Richter GT, Suen JY. Diagnosis and management of hemangiomas and vascular malformations of the head and neck. Oral Dis. 2010;16(5):405-18.

4. Horbach SE, Lokhorst MM, Saeed P, de Goüyon Matignon de Pontouraude CM, Rothová A, van der Horst CM. Sclerotherapy for low-flow vascular malformations of the head and neck: A systematic review of sclerosing agents. J Plast Reconstr Aesthet Surg. 2016;69(3):295-304 .

5. Wassef $M$, Blei $F$, Adams $D$, Alomari $A$, Baselga $E$, Berenstein $A$ et al. Vascular anomalies classification: recommendations from the international society for the study of vascular anomalies. Pediatrics.2015;136(1): e203-14.

6. Li J, Chen J, Zheng G, Liao G, Fu Z, Li J et al. Digital percutaneous sclerotherapy of venous malformations with pingyangmycin and/or absolute ethanol in the maxillofacial region. $J$ Oral MaxillofacSurg 2010;68:2258-266. 
7. Burrows PE, Manson KP. Percutaneus treatment of low flow vascular malformations. J Vasc Interv Radiol. 2004;15:431-45.

8. Ozaki M, Kurita M, Kaji N, Fujino T, Narushima M, Takushima A et al. Efficacy and evaluation of safety of sclerosants for intramuscular venous malformations: clinical and experimental studies. J Plast Surg Hand Surg. 2010;44:75-87.

9. Medeiros SP, Batista AUD, Forte FDS. Prevalência de sintomas de disfunção temporomandibular e hábitos parafuncionais em estudantes universitários. Rev Gauch Odontol. 2011;59(2):201-8.

10. Bortolleto PPB, Moreira APSM, Madureira PR. Analyses parafunctional habits and association with Temporomandibular Disorder. Rev Assoc Paul Cir Dent. 2013;67(3):216-21.

11. Okeson JP. Tratamento das desordens temporomandibulares e oclusão. 6. ed. São Paulo: Elsevier; 2008.

12. Vasconcelos IGS, Venâncio GN, Silva FB. Tratamento de disfunção temporomandibular com placa oclusal: relato de caso. Arch Health Invest. 2018;7(6):205-9.

13. Okeson JP, De Leeuw R. Differential diagnosis of temporomandibular disorders and other orofacial pain disorders. Dent Clin N Am. 2011;55:105-20.

14. Marquezan M, Figueiro C. Alternativa de tratamento de disfunção temporomandibular em um paciente portador de prótese total maxilar e prótese parcial removível mandibular: relato de caso. Rev Dentística Online. 2007;7:77-84.

15. Blanco-Hungría A, Blanco-Aguilera A, BlancoAguilera E, Serrano-del-Rosal R, BiedmaVelázquez L, Rodríguez-Torronteras $A$ et al. Prevalence of the different Axis I clinical subtypes in a sample of patients with orofacial pain and temporomandibular disorders in the Andalusian Healthcare Service. Rev Med Oral Patol Oral Cir Bucal. 2016;21:169-77.

16. Maluf SA, Moreno BGD, Alfredo PP, Amélia Pasqual Marques AP, Rodrigues G. Exercícios terapêuticos nas desordens temporomandibulares: uma revisão de literatura. Fisioter Pesq. 2008;15(4):408-15.

17. Oliveira KB, Pinheiro ICO, Freitas DG, Gualberto HD, Carvalho NAA. A abordagem fisioterapêutica na disfunção da articulação temporomandibular. Revisão da literatura. Med Reabil. 2010;29(3):61-4.

18. Peliciol M, Myra RP, Florianovicz VC, Batista JS. Physiotherapeutic treatment in temporomandibular disorders. Rev Dor. 2017;18(4):355-61.
CONFLITO DE INTERESSES

Os autores declaram não haver conflitos de interesse

AUTOR PARA CORRESPONDÊNCIA

Juliana dos Santos Cativo

Avenida Parintins 154, Cachoeirinha, Manaus-AM, Brasil

E-mail: ju.cativo@gmail.com

Submetido em 04/05/2020 Aceito em 23/10/2020 